\title{
A 2D graphical representation of the sequences of DNA based on triplets and its application
}

\author{
Sai Zou, Lei Wang ${ }^{*}$ and Junfeng Wang
}

\begin{abstract}
In this paper, we first present a new concept of 'weight' for 64 triplets and define a different weight for each kind of triplet. Then, we give a novel 2D graphical representation for DNA sequences, which can transform a DNA sequence into a plot set to facilitate quantitative comparisons of DNA sequences. Thereafter, associating with a newly designed measure of similarity, we introduce a novel approach to make similarities/dissimilarities analysis of DNA sequences. Finally, the applications in similarities/dissimilarities analysis of the complete coding sequences of $\beta$-globin genes of 11 species illustrate the utilities of our newly proposed method.
\end{abstract}

Keywords: Graphical representation; Similarities/dissimilarities analysis; Triplet; DNA sequence

\section{Introduction}

In the recent years, an exponential growth of sequence data in DNA databases has been observed by biologists; the importance of understanding genetic sequences coupled with the difficulty of working with such immense volumes of DNA sequence data underscores the urgent need for supportive visual tools. Recently, graphical representation is well regarded which can offer visual inspection of data and provide a simple way to facilitate the similarity analysis and comparison of DNA sequences [1-5]. Because of its convenience and excellent maneuverability, currently, all kinds of methods based on graphical representation have been extensively applied in relevant realms of bioinformatics.

Until now, there are many different graphical representation methods having been proposed to numerically characterize DNA sequences on the basis of different multiple-dimension spaces. For example, Liao et al. [6-9], Randic et al. [10-13], Guo et al. [14,15], Qi et al. [16], Dai et al. $[17,18]$, and Dorota et al. [19] proposed different 2D graphical representation methods of DNA sequences, respectively. Liao et al. [20-23], Randic et al. [24,25], Qi et al. [26], Yu et al. [27], and Aram et al. [28] proposed different 3D graphical representation methods of DNA sequences, respectively. Liao et al. [29], Tang et al. [30], and Chi et al. [31] proposed different 4D graphical representation

\footnotetext{
* Correspondence: phd.leiwang@gmail.com

School of Software Engineering, Chongqing College of Electronic Engineering, Chongqing 401331, People's Republic of China
}

methods of DNA sequences, respectively. In addition, Liao et al. [32] also proposed a kind of 5D representation method of DNA sequences and so on.

In these approaches mentioned above, most of them adopt the leading eigenvalues of some matrices, such as $L / L$ matrices, $M / M$ matrices, $E$ matrices, covariance matrices, and $D / D$ matrices, to weigh the similarities/ dissimilarities among the complete coding sequences of $\beta$-globin genes of different species. Because the matrix computation is needed to obtain the leading eigenvalues, these methods are usually computationally expensive for long DNA sequences. Furthermore, in some of these approaches, their results of similarities/dissimilarities analysis are not quite reasonable, and there are some results that do not accord with the fact $[7,9]$.

To degrade the computational complexity and obtain more reasonable results of similarities/dissimilarities analysis of DNA sequences, in this article, we propose a new 2D graphical representation of DNA sequences based on triplets, in which, we present a new concept of 'weight' for 64 triplets and a new concept of 'weight deviation' to weigh the similarities/dissimilarities among the complete coding sequences of $\beta$-globin genes of different species. Compared with some existing graphical representations of the DNA sequences, our new scheme has the following advantages: (1) no matrix computation is needed, and (2) it can characterize the graphical representations for DNA sequences exactly and obtain reasonable results of similarities/dissimilarities analysis of DNA sequences. 


\section{Proposed 2D graphical representation of DNA sequence}

Codon is a specific sequence of three adjacent nucleotides on the mRNA that specifies the genetic code information for synthesizing a particular amino acid. As illustrated in Table 1, there are total 20 amino acids and 64 codons in the natural world, and each of these codons has a specific meaning in protein synthesis: 64 codons represent amino acids and the other 3 codons cause the termination of protein synthesis.

For the 64 codons illustrated in Table 1, their corresponding triplets of DNA are illustrated in Table 2.

Based on the above 64 triplets of DNA illustrated in Table 2, we define a new mapping $\Psi$ to map each of these triplets into a different weight. Obviously, the mapping $\Psi$ shall satisfy the following rule: for any two pairs of triplets $\left(X_{1}, Y_{1}\right)$ and $\left(X_{2}, Y_{2}\right)$, where $X_{1}, Y_{1}, X_{2}$, and $Y_{2}$ are all triplets, if the corresponding codons of $X_{1}$ and $Y_{1}$ code the same amino acid but the corresponding codons of $X_{2}$ and $Y_{2}$ code two different amino acids, then there shall be $\left|\Psi\left(X_{1}\right)-\Psi\left(Y_{1}\right)\right|<\left|\Psi\left(X_{2}\right)-\Psi\left(Y_{2}\right)\right|$. So, according to the above rule and for the sake of convenience, weights consist of amino acid and codon. Amino acid is the integer part of weight, and codon is the fractional part of weight. Alanine is defined as 1 , arginine is defined as 2 , and the rest can be done in the same manner. Codons of every amino acid are reordered, so the first codon of alanine's (GCT) weight value is 1.1. We design the detailed mapping rules of $\Psi$ as illustrated in Table 3.

Table 1 Relationship between 20 different kinds of most common amino acids and 64 different kinds of mRNA codons

\begin{tabular}{llll}
\hline Codons & $\begin{array}{l}\text { Amino } \\
\text { acid }\end{array}$ & Codons & Amino acid \\
\hline $\begin{array}{l}\text { GCU, GCC, GCA, } \\
\text { GCG }\end{array}$ & Alanine & $\begin{array}{l}\text { CUU, CUC, CUA, } \\
\text { CUG, UUA, UUG }\end{array}$ & Leucine \\
CGU, CGC, CGA, & Arginine & AAA, AAG & Lysine \\
CGG, AGA, AGG & Aspartic & AUG & Methionine \\
GAU, GAC & acid & & \\
AAU, AAC & Asparagine & UUU, UUC & Phenylalanine \\
UGU, UGC & Cysteine & CCU, CCC, CCA, & Proline \\
GAA, GAG & Glutamic & UCU, UCC, UCA, & Serine \\
& acid & UCG, AGU, AGC & \\
CAA, CAG & Glutamine & ACU, ACC, ACA, ACG & Threonine \\
GGU, GGC, GGA, & Glycine & UGG & Tryptophan \\
GGG & Histidine & UAU, UAC & Tyrosine \\
CAU, CAC & Isoleucine & GUU, GUC, GUA, & Valine \\
AUU, AUC, AUA & & GUG & \\
UAA, UAG, UGA & & &
\end{tabular}

Table 2 The corresponding triplets of 64 codons

\begin{tabular}{|c|c|c|c|}
\hline Codons & $\begin{array}{l}\text { Corresponding } \\
\text { triplets }\end{array}$ & Codons & $\begin{array}{l}\text { Corresponding } \\
\text { triplets }\end{array}$ \\
\hline $\begin{array}{l}\text { GCU, GCC, GCA, } \\
\text { GCG }\end{array}$ & $\begin{array}{l}\text { GCT, GCC, GCA, } \\
\text { GCG }\end{array}$ & $\begin{array}{l}\text { CUU, CUC, } \\
\text { CUA, CUG, } \\
\text { UUA, UUG }\end{array}$ & $\begin{array}{l}\text { CTT, CTC, CTA, } \\
\text { CTG, TTA, TTG }\end{array}$ \\
\hline $\begin{array}{l}\text { CGU, CGC, CGA, } \\
0020 C G G, A G A \\
\text { AGG }\end{array}$ & $\begin{array}{l}C G T, C G C, C G A, \\
C G G, A G A, A G G\end{array}$ & $A A A, A A G$ & AAA, AAG \\
\hline GAU, GAC & GAT, GAC & $A \cup G$ & ATG \\
\hline$A A U, A A C$ & AAT, AAC & UUU, UUC & TाT, TTC \\
\hline UGU, UGC & TGT, TGC & $\begin{array}{l}C C U, C C C, \\
C C A, C C G\end{array}$ & $\begin{array}{l}C C T, C C C, C C A, \\
C C G\end{array}$ \\
\hline GAA, GAG & GAA, GAG & $\begin{array}{l}\text { UCU, UCC, } \\
\text { UCA, UCG, } \\
\text { AGU, AGC }\end{array}$ & $\begin{array}{l}\text { TCT, TCC, TCA, } \\
\text { TCG, AGT, AGC }\end{array}$ \\
\hline CAA, CAG & CAA, CAG & $\begin{array}{l}A C U, A C C \\
A C A, A C G\end{array}$ & $\begin{array}{l}\mathrm{ACT}, \mathrm{ACC}, \mathrm{ACA}, \\
\mathrm{ACG}\end{array}$ \\
\hline $\begin{array}{l}\text { GGU, GGC, GGA, } \\
\text { GGG }\end{array}$ & $\begin{array}{l}\text { GGT, GGC, GGA, } \\
\text { GGG }\end{array}$ & UGG & TGG \\
\hline$C A \cup, C A C$ & CAT, CAC & UAU, UAC & TAT, TAC \\
\hline$A \cup \cup, A \cup C, A \cup A$ & ATT, ATC, ATA & $\begin{array}{l}\text { GUU, GUC, } \\
\text { GUA, GUG }\end{array}$ & $\begin{array}{l}\text { GTT, GTC, GTA, } \\
\text { GTG }\end{array}$ \\
\hline UAA, UAG, UGA & TAA, TAG, TGA & & \\
\hline
\end{tabular}

For example, from Table 3, we will have $\Psi(\mathrm{GCT})=$ $1.1, \Psi(\mathrm{GCC})=1.2, \Psi$ (ATG) $=20.1$, etc., and in addition, we can propose a novel 2D graphical representation of DNA sequences as follows:

Let $G=g_{1}, g_{2}, g_{3} \ldots g_{N}$ be an arbitrary DNA primary sequence, where $g_{i} \in\{\mathrm{A}, \mathrm{T}, \mathrm{G}, \mathrm{C}\}$ for any $i \in\{1,2, \ldots, N\}$, and then, we can transform $G$ into a sequence of triplets such as $G=t_{1}, t_{2}, t_{3} \ldots t_{M}$, where $M=[N / 3]$ and $t_{i}$ is a triplet of DNA for any $i \in\{1,2, \ldots, M\}$. Thereafter, we can define a new mapping $\Theta$ to map $G$ into a plot set as illustrated in the formula (1).

$$
\Theta(G)=\left\{\left(1, \Psi\left(t_{1}\right)\right),\left(2, \Psi\left(t_{2}\right)\right), \ldots,\left(M, \Psi\left(t_{M}\right)\right)\right\}
$$

As for the complete coding sequences of $\beta$-globin genes of 11 species illustrated in the Table 4, each of them can be mapped into a plot set by using the new given mapping $\Theta$, and the $2 \mathrm{D}$ graphical representations corresponding to the complete coding sequences of $\beta$ globin genes of human, chimpanzee, and opossum are shown in Figures 1, 2, and 3, respectively.

\section{Similarity analysis of DNA sequence}

Let $G=g_{1}, g_{2}, g_{3} \ldots g_{N}$ be an arbitrary complete coding sequence, where $g_{i} \in\{\mathrm{A}, \mathrm{T}, \mathrm{G}, \mathrm{C}\}$ for any $i \in\{1,2, \ldots, N\}$, and $G=t_{1}, t_{2}, t_{3} \ldots t_{M}$ be its corresponding sequence of triplets, where $M=[N / 3]$ and $t_{i}$ is a triplet of DNA for any $i \in\{1,2, \ldots, M\}$. Then, we define a function $\delta$ and let $\delta\left(t_{i}\right)$ represent the total number of times that the triplet 
Table 3 The mapping rules of $\psi$

\begin{tabular}{|c|c|c|c|}
\hline Triplet & Corresponding weight & Triplet & Corresponding weight \\
\hline$\overline{\mathrm{GCT}}$ & 1.1 & $\mathrm{CTT}$ & 11.1 \\
\hline GCC & 1.2 & CTC & 11.2 \\
\hline GCA & 1.3 & CTA & 11.3 \\
\hline \multirow[t]{3}{*}{ GCG } & 1.4 & CTG & 11.4 \\
\hline & & TTA & 11.5 \\
\hline & & $\pi G$ & 11.6 \\
\hline CGT & 2.1 & AAA & 12.3 \\
\hline CGC & 2.2 & AAG & 12.4 \\
\hline CGA & 2.3 & & \\
\hline CGG & 2.4 & & \\
\hline AGA & 2.5 & & \\
\hline AGG & 2.6 & & \\
\hline GAT & 3.3 & $\pi T$ & 13.1 \\
\hline GAC & 3.4 & $\pi C$ & 13.2 \\
\hline AAT & 4.1 & $\mathrm{CCT}$ & 14.1 \\
\hline \multirow[t]{3}{*}{ AAC } & 4.2 & CCC & 14.2 \\
\hline & & CCA & 14.3 \\
\hline & & CCG & 14.4 \\
\hline TGT & 5.1 & $\mathrm{TCT}$ & 15.1 \\
\hline \multirow[t]{5}{*}{ TGC } & 5.2 & TCC & 15.2 \\
\hline & & TCA & 15.3 \\
\hline & & TCG & 15.4 \\
\hline & & AGT & 15.5 \\
\hline & & AGC & 15.6 \\
\hline GAA & 6.1 & $\mathrm{ACT}$ & 16.3 \\
\hline \multirow[t]{3}{*}{ GAG } & 6.2 & ACC & 16.4 \\
\hline & & $\mathrm{ACA}$ & 16.5 \\
\hline & & ACG & 16.6 \\
\hline CAA & 7.1 & TGG & 17.3 \\
\hline CAG & 7.2 & & \\
\hline GGT & 8.1 & TAT & 18.1 \\
\hline GGC & 8.2 & TAC & 18.2 \\
\hline GGA & 8.3 & & \\
\hline GGG & 8.4 & & \\
\hline CAT & 9.1 & $\mathrm{GTT}$ & 19.1 \\
\hline \multirow[t]{3}{*}{ CAC } & 9.2 & GTC & 19.2 \\
\hline & & GTA & 19.3 \\
\hline & & GTG & 19.4 \\
\hline ATT & 10.1 & ATG & 20.1 \\
\hline ATC & 10.2 & & \\
\hline ATA & 10.3 & & \\
\hline TAA & 21.1 & & \\
\hline TAG & 21.2 & & \\
\hline TGA & 21.3 & & \\
\hline
\end{tabular}

$t_{i}$ repeats in the sequence of triplets $G=t_{1}, t_{2}, t_{3} \ldots t_{M}$ for any $i \in\{1,2, \ldots, M\}$.

Let $T_{1}=\mathrm{GCT}, T_{2}=\mathrm{GCC}, T_{3}=\mathrm{GCA}, T_{4}=\mathrm{GCG}, T_{5}=$ CGT, $T_{6}=\mathrm{CGC}, T_{7}=\mathrm{CGA}, T_{8}=\mathrm{CGG}, T_{9}=\mathrm{AGA}, T_{10}=$ AGG, $T_{11}=\mathrm{GAT}, T_{12}=\mathrm{GAC}, T_{13}=\mathrm{AAT}, T_{14}=\mathrm{AAC}$, $T_{15}=\mathrm{TGT}, T_{16}=\mathrm{TGC}, T_{17}=\mathrm{GAA}, T_{18}=\mathrm{GAG}, T_{19}=$ CAA, $T_{20}=$ CAG, $T_{21}=$ GGT, $T_{22}=$ GGC,$T_{23}=$ GGA, $T_{24}=\mathrm{GGG}, \quad T_{25}=\mathrm{CAT}, \quad T_{26}=\mathrm{CAC}, \quad T_{27}=\mathrm{ATT}, \quad T_{28}=$ ATC $, \quad T_{29}=\mathrm{ATA}, \quad T_{30}=\mathrm{CTT} T_{31}=\mathrm{CTC}, T_{32}=\mathrm{CTA}$, $T_{33}=\mathrm{CTG}, \quad T_{34}=\mathrm{TTA}, \quad T_{35}=\mathrm{TTG}, T_{36}=\mathrm{AAA}, T_{37}=$ AAG, $T_{38}=$ TTT,$\quad T_{39}=$ TTC $, T_{40}=\mathrm{CCT}, T_{41}=\mathrm{CCC}$, $T_{42}=\mathrm{CCA}, T_{43}=\mathrm{CCG}, T_{44}=\mathrm{TCT}, T_{45}=\mathrm{TCC}, T_{46}=\mathrm{TCA}$, $T_{47}=\mathrm{TCG}, T_{48}=\mathrm{AGT}, T_{49}=\mathrm{AGC}, T_{50}=\mathrm{ACT}, T_{51}=$ ACC, $T_{52}=\mathrm{ACA}, T_{53}=\mathrm{ACG}, T_{54}=\mathrm{TGG}, T_{55}=\mathrm{TAT}$, $T_{56}=$ TAC, $T_{57}=$ GTT, $T_{58}=$ GTC, $T_{59}=$ GTA, $T_{60}=$ GTG, $T_{61}=$ ATG, $T_{62}=$ TAA, $T_{63}=$ TAG , and $T_{64}=$ TGA.

Thereafter, according to Table 2 , since there are a total of 64 triplets of DNA, then we can construct a set of 64 vectors $\left\{<T_{1}, \delta\left(T_{1}\right)>,<T_{2}, \delta\left(T_{2}\right)>, \ldots,<T_{64}, \delta\left(T_{64}\right)>\right\}$ for the given sequence of triplets $G=t_{1}, t_{2}, t_{3} \ldots t_{M}$ as follows: if $T_{i}=t_{j} \in\left\{t_{1}, t_{2}, t_{3}, \ldots t_{M}\right\}$, then $\delta\left(T_{i}\right)=\delta\left(t_{j}\right)$, else $\delta\left(T_{i}\right)=0$, for any $i \in\{1,2, \ldots, 64\}$ and $j \in\{1,2, \ldots, M\}$.

For convenience, we call $\left\{\left\langle T_{1}, \delta\left(T_{1}\right)\right\rangle,\left\langle T_{2}, \delta\left(T_{2}\right)\right\rangle, \ldots\right.$, $\left.<T_{64}, \delta\left(T_{64}\right)>\right\}$ as the triplet-repeat model set of $G$.

For any two given complete coding sequences $A$ and $B$, suppose that their triplet-repeat model sets are $\left\{<T_{1}, X_{1}>,<T_{2}, X_{2}>, \ldots,<T_{64}, X_{64}>\right\}$ and $\left\{<T_{1}, Y_{1}>,<T_{2}\right.$, $\left.Y_{2}>, \ldots,<T_{64}, Y_{64}>\right\}$, respectively. Then, on the basis of the $2 \mathrm{D}$ graphical representation given in the previous Section 2 , we can define the weight deviation between the two DNA sequences $A$ and $B$ as the following formula (2) to measure the similarity between $A$ and $B$.

$$
\mathrm{WD}(A, B)=\frac{\sum_{i=1}^{64}\left|X_{i}-Y_{i}\right| * \Psi\left(T_{i}\right)}{64}
$$

Obviously, the above formula (2) satisfies the fact that the smaller the weight deviation between the two DNA sequences $A$ and $B$, the higher the degree of similarity of $A$ and $B$. According to formula (2), the detailed similarity/dissimilarity matrix obtained for the coding sequences listed in Table 4 is illustrated in Table 5. Basing on the similarity matrix (Table 5 ) constructs a phylogenetic tree, which is shown in Figure 4.

Observing Table 5, it is easy to find out that human, gorilla, and chimpanzee are most similar to each other, and the pairs like gorilla-chimpanzee (with weight deviation of 1.1266), human-gorilla (with weight deviation of 4.3359), and human-chimpanzee (with weight deviation of 5.2500) are the most similar species pairs, but Gallus and opossum are the most dissimilar to the others (with weight deviation bigger than 11). It is consistent with the fact that Gallus is not a mammal, whereas the others 
Table 4 The complete coding sequences of $\beta$-globin genes of 11 species

\section{Species Complete coding sequence}

Human ATGGTGCACCTGACTCCTGAGGAGAAGTCTGCCGTTACTGCCCTGTGGGGCAAGGTGAACGTGGATGAAGTTGGTGGTGAGGCCCTGGGCAGGCTGCTGGTGGTCTACCCTTGGACCCAGAGGTTCTTGAG TCCTITGGGGATCTGTCCACTCCTGATGCTGTTATGGGCAACCCTAAGGTGAAGGCTCATGGCAAGAAAGTGCTCGGTGCCTITAGTGATGGCCTGGCTCACCTGGACAACCTCAAGGGCACCTITGCCACACT GAGTGAGCTGCACTGTGACAAGCTGCACGTGGATCCTGAGAACTTCAGGCTCCTGGGCAACGTGCTGGTCTGTGTGCTGGCCCATCACTTTGGCAAAGAATTCACCCCACCAGTGCAGGCTGCCTATCAGAAA GTGGTGGCTGGTGTGGCTAATGCCCTGGCCCACAAGTATCACTAA

Chimpanzee ATGGTGCACCTGACTCCTGAGGAGAAGTCTGCCGTTACTGCCCTGTGGGGCAAGGTGAACGTGGATGAAGTTGGTGGTGAGGCCCTGGGCAGGTTGGTATCAAGGCTGCTGGTGGTCTACCCTTGGACCCAG AGGTTCTITGAGTCCTITGGGGATCTGTCCACTCCTGATGCTGTTATGGGCAACCCTAAGGTGAAGGCTCATGGCAAGAAAGTGCTCGGTGCCTITAGTGATGGCCTGGCTCACCTGGACAACCTCAAGGGCAC CTITGCCACACTGAGTGAGCTGCACTGTGACAAGCTGCACGTGGATCCTGAGAACTTCAGGCTCCTGGGCAACGTGCTGGTCTGTGTGCTGGCCCATCACTTTGGCAAAG

Gorilla ATGGTGCACCTGACTCCTGAGGAGAAGTCTGCCGTTACTGCCCTGTGGGGCAAGGTGAACGTGGATGAAGTTGGTGGTGAGGCCCTGGGCAGGCTGCTGGTGGTCTACCCTTGGACCCAGAGGTTCTTTGAGT CCTTTGGGGATCTGTCCACTCCTGATGCTGTTATGGGCAACCCTAAGGTGAAGGCTCATGGCAAGAAAGTGCTCGGTGCCTTTAGTGATGGCCTGGCTCACCTGGACAACCTCAAGGGCACCTTTGCCACACTG AGTGAGCTGCACTGTGACAAGCTGCACGTGGATCCTGAGAACTTCAAGCTCCTGGGCAATGTGCTGGTCTGTGTGCTGGCCCATCACTTTGGCAAAG

Black lemur ATGACTTTGCTGAGTGCTGAGGAGAATGCTCATGTCACCTCTCTGTGGGGCAAGGTGGATGTAGAGAAAGTTGGTGGCGAGGCCTTGGGCAGGCTGCTGGTCGTCTACCCATGGACCCAGAGGTTCTTCGAGT CCTITGGGGACCTGTCCTCTCCTTCTGCTGTTATGGGGAACCCTAAGGTGAAGGCCCATGGCAAGAAGGTGCTGAGTGCCTTAGTGAAGGTCTGCATCACCTGGACAACCTCAAGGGCACCTTTGCTCAACTG AGTGAGCTGCACTGTGACAAGTTGCACGTGGATCCTCAGAACTTCACTCTCCTGGGCAACGTGCTGGTGGTTGTGCTGGCTGAACACTITGGCAATGCATTCAGCCCGGCGGTGCAGGCTGCCTITCAGAAGG TGGTGGCTGGTGTGGCCAATGCTCTGGCTCACAAGTACCACTGA

Norway rat ATGGTGCACCTAACTGATGCTGAGAAGGCTACTGTTAGTGGCCTGTGGGGAAAGGTGAATGCTGATAATGTTGGCGCTGAGGCCCTGGGCAGGCTGCTGGTTGTCTACCCTTGGACCCAGAGGTACTITCTA AATTTGGGGACCTGTCCTCTGCCTCTGCTATCATGGGTAACCCCCAGGTGAAGGCCCATGGCAAGAAGGTGATAAATGCCTTCAATGATGGCCTGAAACACTTGGACAACCTCAAGGGCACCTITGCTCATC GAGTGAACTCCACTGTGACAAGCTGCATGTGGATCCTGAGAACTTCAGGCTCCTGGGCAATATGATTGTGATTGTGTTGGGCCACCACCTGGGCAAGGAATTCACCCCCTGTGCACAGGCTGCCTTCCAGAA GGTGGTGGCTGGAGTGGCCAGTGCCCTGGCTCACAAGTACCACTAA

House ATGGTGCACCTGACTGATGCTGAGAAGTCTGCTGTCTCTTGCCTGTGGGCAAAGGTGAACCCCGATGAAGTTGGTGGTGAGGCCCTGGGCAGGCTGCTGGTTGTCTACCCTTGGACCCAGCGGTACTTTGATAG CITTGGAGACCTATCCTCTGCCTCTGCTATCATGGGTAATCCCAAGGTGAAGGCCCATGGCAAAAAGGTGATAACTGCCTTIAACGAGGGCCTGAAAAACCTGGACAACCTCAAGGGCACCTITGCCAGCCTC AGTGAGCTCCACTGTGACAAGCTGCATGTGGATCCTGAGAACTTCAGGCTCCTAGGCAATGCGATCGTGATTGTGCTGGGCCACCACCTGGGCAAGGATTTCACCCCTGCTGCACAGGCTGCCTTCCAGAAGG TGGTGGCTGGAGTGGCCACTGCCCTGGCTCACAAGTACCACTAA

Goat ATGCTGACTGCTGAGGAGAAGGCTGCCGTCACCGGCTTCTGGGGCAAGGTGAAAGTGGATGAAGTTGGTGCTGAGGCCCTGGGCAGGCTGCTGGTTGTCTACCCCTGGACTCAGAGGTTCTTGGAGCACTTI GGGGACTTGTCCTCTGCTGATGCTGTTATGAACAATGCTAAGGTGAAGGCCCATGGCAAGAAGGTGCTAGACTCCTITAGTAACGGCATGAAGCATCTTGACGACCTCAAGGGCACCTITGCTCAGCTGAGT GAGCTGCACTGTGATAAGCTGCACGTGGATCCTGAGAACTTCAAGCTCCTGGGCAACGTGCTGGTGGTTGTGCTGGCTCGCCACCATGGCAGTGAATTCACCCCGCTGCTGCAGGCTGAGTTTCAGAAGGTG GTGGCTGGTGTTGCCAATGCCCTGGCCCACAGATATCACTAA

Bovine ATGCTGACTGCTGAGGAGAAGGCTGCCGTCACCGCCTTITGGGGCAAGGTGAAAGTGGATGAAGTTGGTGGTGAGGCCCTGGGCAGGCTGCTGGTTGTCTACCCCTGGACTCAGAGGTTCTTTGAGTCCTTTG GGGACTTGTCCACTGCTGATGCTGTTATGAACAACCCTAAGGTGAAGGCCCATGGCAAGAAGGTGCTAGATTCCTTTAGTAATGGCATGAAGCATCTCGATGACCTCAAGGGCACCTTTGCTGCGCTGAGTGA GCTGCACTGTGATAAGCTGCATGTGGATCCTGAGAACTTCAAGCTCCTGGGCAACGTGCTAGTGGTTGTGCTGGCTCGCAATITGGCAAGGAATTCACCCCGGTGCTGCAGGCTGACTITCAGAAGGTGGTG GCTGGTGTGGCCAATGCCCTGGCCCACAGATATCATTAA

Rabbit ATGGTGCATCTGTCCAGTGAGGAGAAGTCTGCGGTCACTGCCCTGTGGGGCAAGGTGAATGTGGAAGAAGTTGGTGGTGAGGCCCTGGGCAGGCTGCTGGTTGTCTACCCATGGACCCAGAGGTTCTTCGAG TCCTITGGGGACCTGTCCTCTGCAAATGCTGTTATGAACAATCCTAAGGTGAAGGCTCATGGCAAGAAGGTGCTGGCTGCCTTCAGTGAGGGTCTGAGTCACCTGGACAACCTCAAAGGCACCTITGCTAAGCT GAGTGAACTGCACTGTGACAAGCTGCACGTGGATCCTGAGAACTTCAGGCTCCTGGGCAACGTGCTGGTTATTGTGCTGTCTCATCATTTGGCAAAGAATTCACTCCTCAGGTGCAGGCTGCCTATCAGAAG GTGGTGGCTGGTGTGGCCAATGCCCTGGCTCACAAATACCACTGA

Opossum ATGGTGCACTTGACTTCTGAGGAGAAGAACTGCATCACTACCATCTGGTCTAAGGTGCAGGTTGACCAGACTGGTGGTGAGGCCCTTGGCAGGATGCTCGTTGTCTACCCCTGGACCACCAGGTTIITGGGA GCTTTGGTGATCTGTCCTCTCCTGGCGCTGTCATGTCAAATTCTAAGGTTCAAGCCCATGGTGCTAAGGTGTTGACCTCCTTCGGTGAAGCAGTCAAGCATTTGGACAACCTGAAGGGTACTTATGCCAAGTTG AGTGAGCTCCACTGTGACAAGCTGCATGTGGACCCTGAGAACTTCAAGATGCTGGGGAATATCATTGTGATCTGCCTGGCTGAGCACTTTGGCAAGGATIITACTCCTGAATGTCAGGTGGTTGGCAGAAGCT CGTGGCTGGAGTTGCCCATGCCCTGGCCCACAAGTACCACTAA

ATGGTGCACTGGACTGCTGAGGAGAAGCAGCTCATCACCGGCCTCTGGGGCAAGGTCAATGTGGCCGAATGTGGGGCCGAAGCCCTGGCCAGGCTGCTGATCGTCTACCCCTGGACCCAGAGGTTCTITGCG TCCTIGGGACCTCTCCAGCCCCACTGCCATCCTTGGCAACCCCATGGTCCGCGCCCACGGCAAGAAAGTGCTCACCTCCTITGGGGATGCTGTGAAGAACCTGGACAACATCAAGAACACCTTCTCCCAAC TGTCCGAACTGCATTGTGACAAGCTGCATGTGGACCCCGAGAACTTCAGGCTCCTGGGTGACATCCTCATCATTGTCCTGGCCGCCCACTTCAGCAAGGACTTCACTCCTGAATGCCAGGCTGCCTGGCAGAA GCTGGTCCGCGTGGTGGCCCATGCCCTGGCTCGCAAGTACCACTAA 


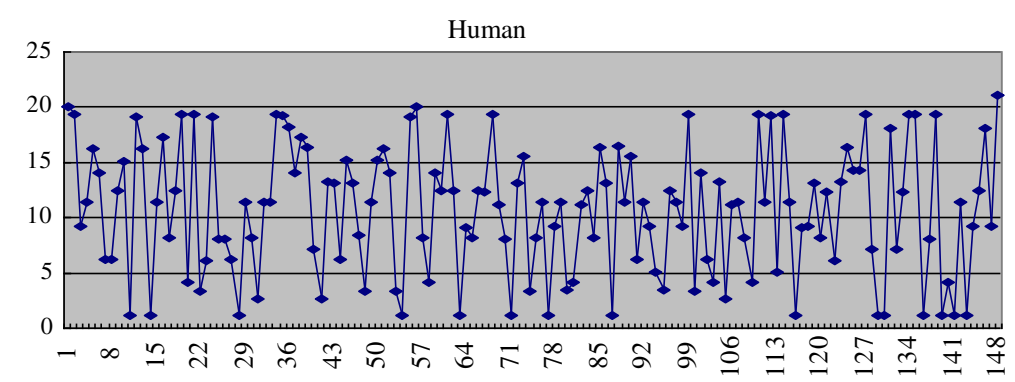

Figure 1 The 2D graphical representations of the complete coding sequences of $\beta$-globin genes of human.

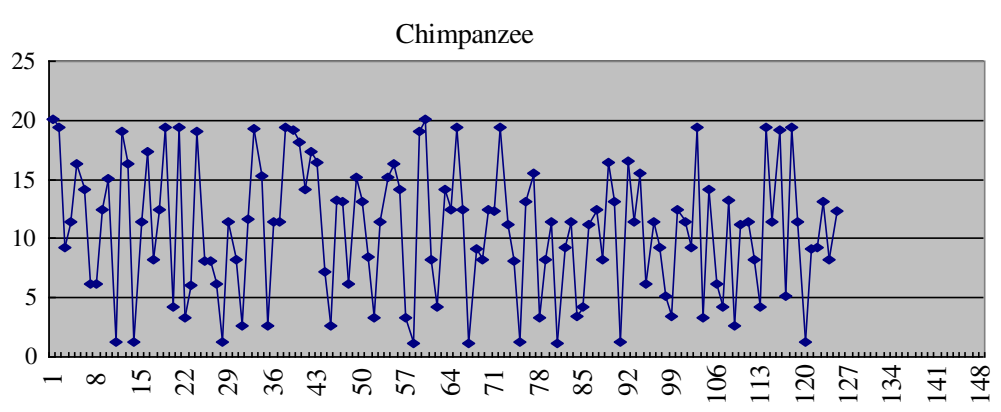

Figure 2 The 2D graphical representations of the complete coding sequences of $\beta$-globin genes of chimpanzee.

are mammals, and opossum is the most remote species from the remaining mammals. Similar results have been obtained in other papers by different approaches $[2,5,7,9,33]$.

For testing the validity of our method, the existing results of the examination of the degree of similarity/ dissimilarity of the coding sequences of $\beta$-globin genes of several species with the coding sequence of the human $\beta$ globin gene by means of approaches using alternative DNA sequence descriptors $[2,5,7,9]$ are listed in Table 6 for comparison.

From Table 6, we can find that the pairs like humangorilla and human-chimpanzee are the two most similar species pairs when adopting (A) the method of our work, (B) the method of [2], (C) the method of [5], and
(D) the method of [7], which is in accordance with the fact that gorilla and chimpanzee are the two most closest species of human, but when adopting (E) the method of [9], the most similar species pair is human-goat, which is obviously not correct. In addition, the pairs like human-Gallus and human-opossum are the two most dissimilar species pairs when adopting (A) the method of our work, (C) the method of [5], and (E) the method of [9], which is in accordance with the fact that Gallus is not a mammal, whereas the others are mammals, and opossum is the most remote species from the remaining mammals. However, when adopting (D) the method of [7], the two most dissimilar species pairs are humanopossum and human-lemur, which is obviously not reasonable also.

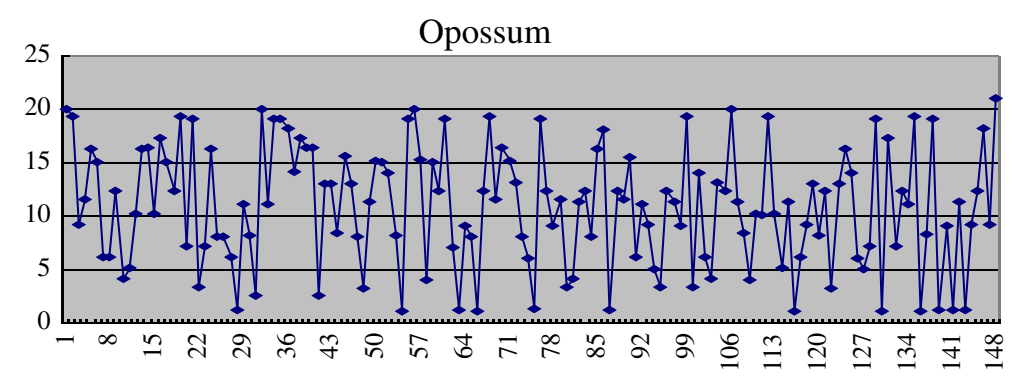

Figure 3 The 2D graphical representations of the complete coding sequences of $\beta$-globin genes of opossum. 
Table 5 The similarity/dissimilarity matrix for the coding sequences of Table 1 based on the weight deviation

\begin{tabular}{|c|c|c|c|c|c|c|c|c|c|c|c|}
\hline & Human & Chimpanzee & Gorilla & Lemur & Rat & Mouse & Goat & Bovine & Rabbit & Opossum & Gallus \\
\hline Human & 0 & 5.2500 & 4.3359 & 8.5891 & 10.670 & 9.7047 & 8.2219 & 8.1438 & 7.8281 & 15.6078 & 16.7109 \\
\hline Chimpanzee & & 0 & 1.1266 & 8.0297 & 10.645 & 9.6016 & 8.4375 & 9.3219 & 9.6000 & 14.2578 & 15.8734 \\
\hline Gorilla & & & 0 & 7.8688 & 9.9625 & 8.6063 & 7.6734 & 8.5578 & 8.5547 & 13.9719 & 14.8781 \\
\hline Lemur & & & & 0 & 8.7219 & 9.5500 & 7.1328 & 9.3891 & 5.6891 & 12.9281 & 15.2000 \\
\hline Rat & & & & & 0 & 6.0750 & 7.0484 & 9.3641 & 9.6578 & 13.5906 & 14.1219 \\
\hline Mouse & & & & & & 0 & 9.4953 & 9.2641 & 10.7984 & 12.3406 & 12.3688 \\
\hline Goat & & & & & & & 0 & 5.2625 & 8.7219 & 11.9703 & 14.5359 \\
\hline Bovine & & & & & & & & 0 & 9.2906 & 12.5922 & 15.0234 \\
\hline Rabbit & & & & & & & & & 0 & 14.8984 & 15.6953 \\
\hline Opossum & & & & & & & & & & 0 & 14.2750 \\
\hline Gallus & & & & & & & & & & & 0 \\
\hline
\end{tabular}

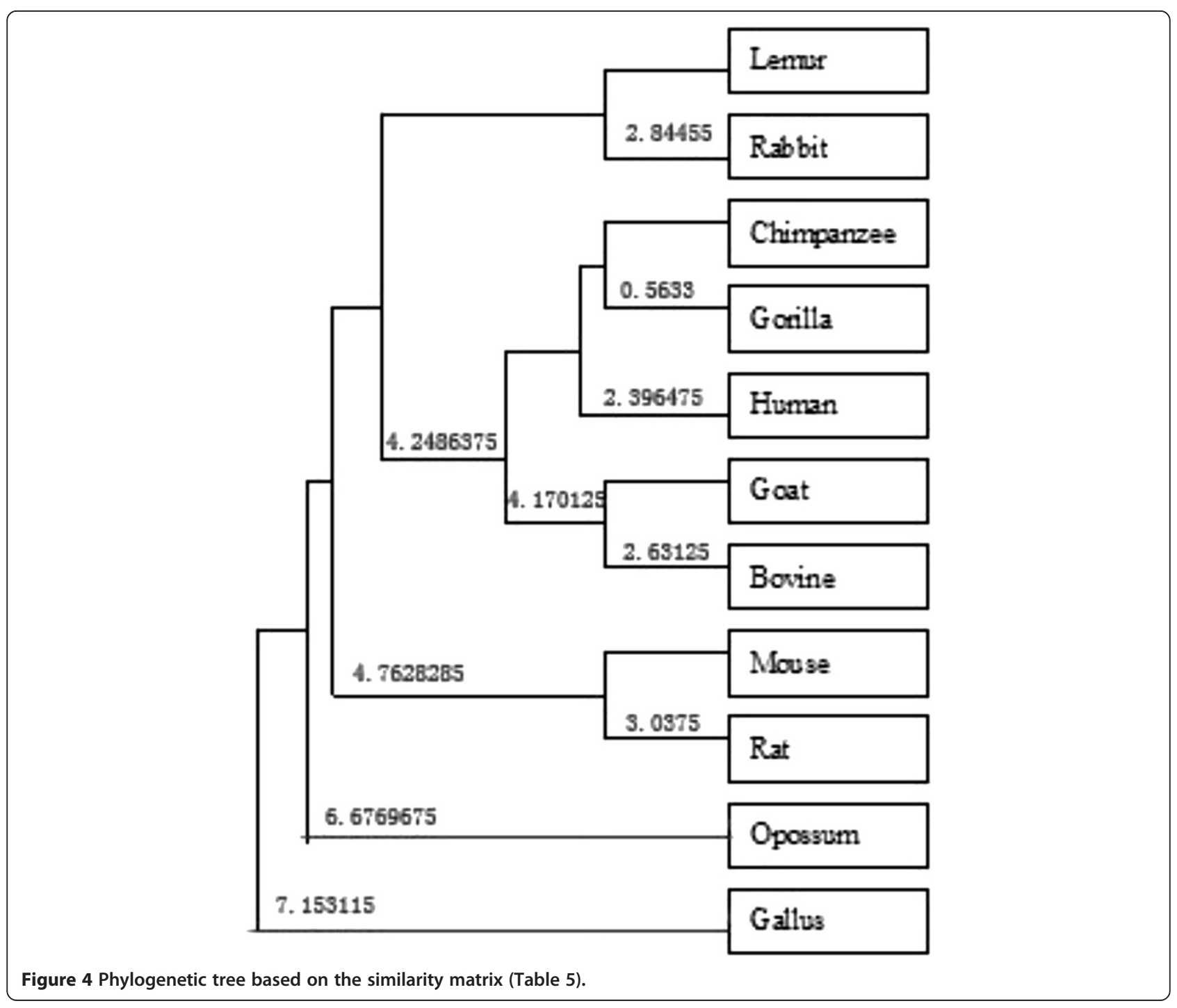


Table 6 The similarity/dissimilarity of the coding sequences

\begin{tabular}{lccccc}
\hline Species & $\mathbf{A}$ & $\mathbf{B}$ & $\mathbf{C}$ & $\mathbf{D}$ & $\mathbf{E}$ \\
\hline Chimpanzee & 5.2500 & 0.0144 & 14.00 & 0.005069 & 0.863 \\
Gorilla & 4.3359 & 0.0125 & 13.63 & 0.006611 & 0.339 \\
Lemur & 8.5891 & - & 31.75 & 0.030894 & 1.188 \\
Rat & 10.670 & 0.1377 & 41.65 & 0.015539 & 1.966 \\
Mouse & 9.7047 & 0.1427 & 30.27 & 0.015700 & 0.735 \\
Goat & 8.2219 & 0.1161 & 31.39 & 0.020980 & 0.311 \\
Bovine & 8.1438 & 0.0773 & 30.68 & 0.017700 & 2.489 \\
Rabbit & 7.8281 & 0.1332 & 35.575 & 0.015788 & 1.372 \\
Opossum & 15.6078 & - & 48.701 & 0.033363 & 6.322 \\
Gallus & 16.7109 & - & 70.46 & 0.025801 & 7.170 \\
\hline
\end{tabular}

\section{Conclusion}

In this paper, we propose a new $2 \mathrm{D}$ graphical representation for DNA sequences based on triplets, and associating with a newly introduced concept of weight of triplets and a newly designed measure of similarity named weight deviation, we propose a new method to make similarity analysis of DNA sequences, in which no matrix computation is needed and reasonable and useful approaches for both computational scientists and molecular biologists to effectively analyze DNA sequences can be provided at the same time.

\section{Competing interests}

The authors declare that they have no competing interests.

\section{Acknowledgements}

This work is supported by the Chongqing Education Science Project of China in 2014, Chongqing "Twelfth Five Year plan" educational programming projects of China (2013-ZJ-077), program for university youth backbone teachers of Chongqing in 2014.

Received: 17 August 2013 Accepted: 10 December 2013 Published: 2 January 2014

\section{References}

1. W Chen, B Liao, Y Liu, W Zhu, Z Su, A numerical representation of DNA sequences and its applications. MATCH: Commun Math Comput Chem. 60, 291-300 (2008)

2. N Jafarzadeh, A Iranmanesh, A novel graphical and numerical representation for analyzing DNA sequences based on codons. MATCH: Commun Math Comput Chem. 68, 611-620 (2012)

3. B Liao, BY Liao, XM Sun, QG Zeng, A novel method for similarity analysis and protein sub-cellular localization prediction. Bioinformatics 26, 2678-2683 (2010)

4. XQ Qi, Q Wu, Y Zhang, E Fuller, CQ Zhang, A novel model for DNA sequence similarity analysis based on graph theory. J Evol Bioinform 7. 149-158 (2011)

5. JF Yu, JH Wang, X Sun, Analysis of similarities/dissimilarities of DNA sequences based on a novel graphical representation. MATCH: Commun Math Comput Chem. 63, 493-512 (2010)

6. Y Li, G Huang, B Liao, Z Liu, H-L curve: a novel 2D graphical representation of protein sequences. MATCH: Commun Math Comput Chem. 61, 519-532 (2009)

7. B Liao, TM Wang, New 2D graphical representation of DNA sequences. J. Comput. Chem. 25, 1364-1368 (2004)

8. B Liao, XY Xiang, W Zhu, Coronavirus phylogeny based on 2D graphical representation of DNA sequence. J. Comput. Chem. 27, 1196-1202 (2006)
9. ZB Liu, B Liao, W Zhu, GH Huang, A 2D graphical representation of DNA sequence based on dual nucleotides and its application. Int. J. Quantum Chem. 109, 948-958 (2009)

10. M Randic, M Vracko, J Zupan, M Novic, Compact 2D graphica representation of DNA. Chem. Phys. Lett. 373, 558-562 (2003)

11. M Randic, M Vracko, N Lers, D Plavsic, Analysis of similarity/dissimilarity of 2D graphical representation. Chem. Phys. Lett. 371, 202-207 (2003)

12. M Randic, M Vracko, N Lers, D Plavsic, Novel 2-D graphical representation of DNA sequences and their numerical characterization. Chem. Phys. Lett 368, 1-6 (2003)

13. M Randic, Graphical representations of DNA as 2-D map. Chem. Phys. Lett. 386, 468-471 (2004)

14. XF Guo, M Randic, SC Basak, A novel 2-D graphical representation of DNA sequences of low degeneracy. Chem. Phys. Lett. 350, 106-112 (2001)

15. XF Guo, A Nandy, Numerical characterization of DNA sequences in a 2-D graphical representation scheme of low degeneracy. Chem. Phys. Lett. 369, 361-366 (2003)

16. ZH Qi, XQ Qi, Novel 2D graphical representation of DNA sequence based on dual nucleotides. Chem Phys Lett. 440, 139-144 (2007)

17. Q Dai, ZL Xiu, TM Wang, A novel 2D graphical representation of DNA sequences and its application. J Mol Graph Model. 25, 340-344 (2006)

18. XQ Liu, Q Dai, ZL Xiu, TM Wang, PNN-curve: a new 2D graphical representation of DNA sequences and its application. J. Theor. Biol. 243, 555-561 (2006)

19. BW Dorota, C Timothy, W Piotr, 2D-dynamic representation of DNA sequences. Chem. Phys. Lett. 442, 140-144 (2007)

20. CX Yuan, B Liao, TM Wang, New 3D graphical representation of DNA sequences and their numerical characterization. Chem. Phys. Lett 379, 412-417 (2003)

21. B Liao, TM Wang, 3-D graphical representation of DNA sequences and their numerical characterization. J. Mol. Struct. (THEOCHEM) 681, 209-212 (2004)

22. B Liao, TM Wang, A 3D graphical representation of RNA secondary structure. J Biomol Struct Dynam. 21, 827-832 (2004)

23. Z Cao, B Liao, RF Li, A group of 3D graphical representation of DNA sequences based on dual nucleotides. Int. J. Quantum Chem. 108, 1485-1490 (2008)

24. M Randic, M Vracko, A Nandy, SC Basak, On 3D graphical representation of DNA primary sequences and their numerical characterization. J. Chem. Inf. Comput. Sci. 40, 1235-1244 (2000)

25. M Randic, J Zupan, M Novic, On 3D graphical representation of proteomics maps and their numerical characterization. J. Chem. Inf. Comput. Sci. 41, 1339-1344 (2001)

26. XQ Qi, TR Fan, PN-curve: a 3D graphical representation of DNA sequences and their numerical characterization. Chem. Phys. Lett. 442, 434-440 (2007)

27. JF Yu, X Sun, JH Wang, TN curve: a novel 3D graphical representation of DNA sequence based on trinucleotides and its applications. J. Theor. Biol. 261, 459-468 (2009)

28. $V$ Aram, A Iranmanesh, 3D-dynamic representation of DNA sequences. MATCH: Commun Math Comput Chem. 67, 809-816 (2012)

29. B Liao, MS Tan, KQ Ding, A 4D representation of DNA sequences and its application. Chem. Phys. Lett. 402, 380-383 (2005)

30. XC Tang, PP Zhou, WY Qiu, On the similarity/dissimilarity of DNA sequences based on 4D graphical representation. Chin. Sci. Bull. 55, 701-704 (2010)

31. R Chi, KQ Ding, Novel 4D numerical representation of DNA sequences. Chem. Phys. Lett. 407, 63-67 (2005)

32. B Liao, XY Xiang, RF Li, W Zhu, On the similarity of DNA primary sequences based on 5D representation. J. Math. Chem. 42, 47-57 (2007)

33. $\mathrm{PHe}, \mathrm{J}$ Wang, Characteristic sequences for DNA primary sequence. J. Chem. Inf. Comput. Sci. 42, 1080-1085 (2002)

\section{doi:10.1186/1687-4153-2014-1}

Cite this article as: Zou et al:: A 2D graphical representation of the sequences of DNA based on triplets and its application. EURASIP Journal on Bioinformatics and Systems Biology 2014 2014:1. 